\title{
Field Conjugation Adaptive Arrays in Free-Space Coherent Laser Communications
}

\author{
Aniceto Belmonte and Joseph M. Kahn
}

\begin{abstract}
We analyze the performance of adaptive field conjugation array receivers in coherent laser communications through the turbulent atmosphere. We consider coherent fiber arrays consisting of densely packed multiple subapertures, with each subaperture interfaced to a single-mode fiber. In a field conjugation fiber array, the single-mode fiber outputs are detected, and the photocurrents are adaptively co-phased and scaled, and then summed to mitigate signal fading associated with atmospheric turbulence and compensate for imperfect fiber coupling efficiency. We quantify how field conjugation processing improves performance in the presence of turbulence, as compared to a monolithic-aperture coherent receiver having an equal total cross-sectional area.
\end{abstract}

Index Terms-Atmospheric turbulence; Coherent receivers; Diversity combining; Optical communications.

\section{INTRODUCTION}

$\mathbf{R}$ ecent advances in coherent optical communications in fiber transmission systems [1] have stimulated interest in applying coherent detection in free-space laser communications (see, e.g., [2-9]). In a coherent system, transmitted information can be encoded in the complex electric field, including amplitude, phase and polarization. A coherent receiver measures these degrees of freedom by interfering the received signal with a local oscillator. In a free-space coherent system, atmospheric turbulence can reduce the coherence of the received signal that is to be mixed with the local oscillator. Light propagated through a turbulent atmosphere contains speckle which will be present at the detector surface. Therefore, illuminating a single-element detector with a uniform LO beam will produce mismatch of the amplitudes and phases of the two fields, resulting in a loss of downconverted power [2-5]. The downconverted coherent power is maximized when the spatial field of the received signal matches that of the local oscillator $[10,11]$.

System configurations based on single monolithic apertures using adaptive optical phase compensation to mitigate atmospheric turbulence are being investigated [4]. As an

Manuscript received July 20, 2011; revised September 20, 2011; accepted September 20, 2011; published October 14, 2011 (Doc. ID 151472).

Aniceto Belmonte (e-mail: belmonte@tsc.upc.edu) is with the Technical University of Catalonia, BarcelonaTech, Department of Signal Theory and Communications, 08034 Barcelona, Spain.

Joseph M. Kahn is with Stanford University, E. L. Ginzton Laboratory, Department of Electrical Engineering, Stanford, California 94305, USA.

Digital Object Identifier 10.1364/JOCN.3.000830 alternative, diversity combining techniques, where signals detected by two or more apertures are combined to reduce the probability of deep fades and improve detection efficiency, are being studied [6,7]. If these apertures are sufficiently separated, fades in different apertures can be considered statistically independent.

In this paper, we consider a coherent fiber array consisting of densely packed multiple subapertures, with each subaperture interfaced to a single-mode fiber, for improving the performance of an atmospheric coherent system. Instead of using a single monolithic-aperture coherent receiver with a full-size collecting area, a large effective aperture is achieved by combining the output signals from an array of smaller fiber-coupled subapertures in a close-packed arrangement (see Fig. 1). A coherent fiber array offers an advantage in terms of coupling efficiency, as the number of turbulence speckles over each subaperture is much smaller than it would be over a single large aperture. Now, each receiver aperture can be smaller than the scale on which the signal wavefront varies and the local oscillator phase can be matched to the signal to achieve efficient coherent downconversion. Output signals from these receivers can then be combined electronically to improve the detection statistics. In general, the performance of such a field conjugation adaptive array should improve with an increasing number of subapertures and, given a fixed collecting area, the fiber array system can offer superior performance. Note that, due to the close spatial arrangement of the subapertures in a coherent fiber array, the atmospheric fading on the array components is correlated or dependent.

In this paper, we consider a general model for the output signal to noise ratio (SNR) of a coherent fiber array. In Section II, we define a statistical model to describe the signal collected by the fiber array receiver after propagation through the atmosphere. In Section III, we analyze the performance of array receivers under the effects of log-normal amplitude fluctuations and Gaussian phase fluctuations, in addition to local oscillator shot noise. We present our conclusions in Section IV.

\section{RECEIVER MODEL}

In a coherent communication receiver, the SNR $\gamma_{0}$ per unit bandwidth $B$ for a quantum or shot-noise-limited signal can be interpreted as the detected number of photons (photocounts) per symbol, where $1 / B$ is the symbol period. Coherently detected signals are modeled as narrowband RF signals with 

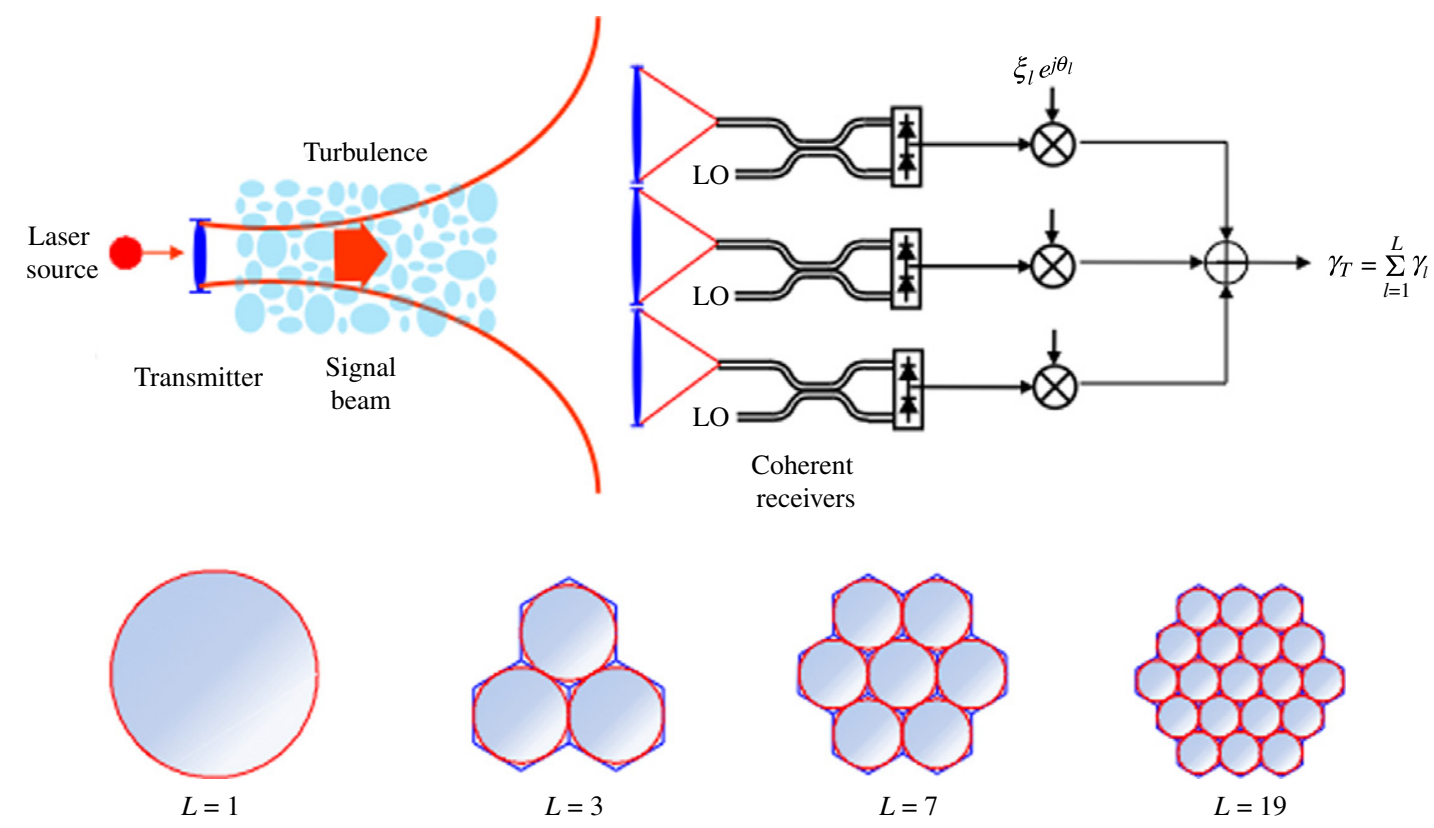

Fig. 1. (Color online) A coherent free-space optical communication system is affected by atmospheric turbulence in several ways. Amplitude scintillation and phase distortion in the receiver plane lead to fluctuations in the amplitude and phase of the detected electrical signal, impairing communication system performance. Adaptive receiver arrays are considered to mitigate the impact of atmospheric turbulence. In a field conjugation fiber array, individual single-mode fiber outputs are detected, and the resulting intermediate-frequency signals are adaptively co-phased and scaled and then summed, thus mitigating amplitude and phase fluctuations caused by turbulence. We analyze coherent fiber arrays composed of $L$ subapertures arranged in a hexagonal close-packed array.

additive white Gaussian noise (AWGN). For a coherent receiver system, in the presence of target speckle and atmospheric turbulence, we must consider fading, in which the signals are also affected by multiplicative noise. In a fading AWGN channel, we let $\alpha^{2}$ denote the atmospheric channel power fading and $\gamma_{0} \alpha^{2}$ denote the instantaneous received SNR per pulse. For a shot-noise-limited coherent optical receiver, the SNR of the envelope detector can be taken as the number of signal photons detected on the receiver aperture $\gamma_{0}$ multiplied by a photo-mixing efficiency $\alpha^{2}$. For systems with perfect spatial mode matching, the mixing efficiency is unity. When the spatial modes are not properly matched, the contributions to the photocurrent from different parts of the receiver aperture can interfere destructively, reducing the instantaneous photo-mixing efficiency, thus causing fading. Note that, conditional on a realization of the atmospheric channel described by $\alpha^{2}$, this is an AWGN channel with instantaneous received SNR $\gamma=\gamma_{0} \alpha^{2}$. This quantity is a function of the random channel power fading $\alpha^{2}$, and is therefore random. The statistical properties of the atmospheric random channel fade $\alpha$, with probability density function (PDF) $P_{\alpha}(\alpha)$, provide a statistical characterization of the SNR $\gamma=\gamma_{0} \alpha^{2}$. In this study we define a statistical model for the fading amplitude $\alpha$ (i.e., SNR $\gamma$ ) of the received signal after propagation through the atmosphere.

In a single aperture, fiber-based coherent receiver, when the spatial field of the received signal $E_{i}(\mathbf{r})$ does not match that of the local oscillator $E_{m}(\mathbf{r})$, as described by the fiber-mode profile referred to the receiving aperture, the random fading

$$
\alpha=\frac{4}{\pi D^{2}} \int d \mathbf{r} W(\mathbf{r}) E_{i}(\mathbf{r}) E_{m}(\mathbf{r})
$$

depends on the amplitude and phase mismatches of the two fields incident on the receiving aperture. Field mismatches are caused by atmospheric turbulence. The circular receiving aperture of diameter $D$ is defined by the aperture function $W(\mathbf{r})$, which equals unity for $|\mathbf{r}| \leq D / 2$, and equals zero for $|\mathbf{r}|>D / 2$. Throughout this analysis, we assume that the signal and local oscillator are matched in polarization, which is not difficult to achieve in practice, owing to the low birefringence of the atmosphere.

In general, in Eq. (1) fading is described by a complex amplitude $\alpha=\alpha_{r}+j \alpha_{i}$, where $\alpha_{r}$ and $\alpha_{i}$ represent integrals over the collecting aperture of the real and imaginary parts, respectively, of the optical fields reaching the receiver. These real and imaginary parts can be considered as the components of a complex random phasor. We need to study how turbulence-induced fluctuations of the optical field define the statistics of the fading amplitude $\alpha=\alpha_{r}+j \alpha_{i}$. From Eq. (1), we note that the two random magnitudes $\alpha_{r}$ and $\alpha_{i}$ can be expressed as integrals over the aperture and, hence, are the sums of contributions from each point in the aperture. In order to proceed with the analysis, we can consider a statistical model in which these continuous integrals are expressed as finite sums over statistically independent cells in the aperture. Under the assumption that the number of independent coherent regions is large enough, we can consider that $\alpha_{r}$ and $\alpha_{i}$ asymptotically approach jointly normal random variables. Then, the probability density function of the fading amplitude $\alpha$ can be well approximated by a Rayleigh distribution. Just as in a speckle pattern [12], the Rayleigh distribution for the turbulence-induced fading amplitude is a consequence of the central-limit theorem. However, under 
conditions of weak turbulence, in which the number of coherent cells is small, the fading may actually be the result of summing a small number of terms. In this case, the fading $\alpha$ is not likely to be Rayleigh. Rather than assuming that $\alpha$ is always Rayleigh distributed for all conditions of turbulence, it is more realistic to assume that $\alpha$ is described by a generalized Rayleigh distribution that becomes Rayleigh only when the number of coherent terms $N$ becomes large enough. Such a distribution is the Nakagami- $m$ distribution [13], in essence, a central chi-square distribution described by

$$
p_{\alpha}(\alpha)=2(m N)^{m} \frac{\alpha^{2 m-1}}{\Gamma(m)} \exp \left(-m N \alpha^{2}\right)
$$

where $\Gamma$ is the complete gamma function. The Nakagami- $m$ parameter $m$ and fading parameter $N$ are measures of turbulence effects. Here, $N$ is the inverse of the fading mean-square value,

$$
N=\frac{1}{\overline{\alpha^{2}}}
$$

and describes the number of field coherent areas affecting the fading measurement [12]. When $m \rightarrow 1$, the $m$-distribution reduces to Rayleigh. Note that the Nakagami- $m$ distribution closely approximates the Rice distribution in most practical situations and there is an exact equivalency between the $m$ parameter and the Rician $K$ factor [13]. We have previously used the Rice distribution to model the impact of atmospheric turbulence-induced fading on free-space optical communication links using coherent detection [7]. Monte Carlo simulations have validated the underlying statistical model [14].

Applying the Jacobian of the transformation $\alpha^{2}=\gamma / \gamma_{0}$, the corresponding SNR $\gamma$ distribution can be described according to a gamma distribution with a shape parameter $m$ and a mean value $\bar{\gamma}=\gamma_{0} \overline{\alpha^{2}} \equiv \gamma_{0} / N$ given by

$$
p_{\gamma}(\gamma)=\left(\frac{m N}{\gamma_{0}}\right)^{m} \frac{\gamma^{m-1}}{\Gamma(m)} \exp \left(-\frac{m N}{\gamma_{0}} \gamma\right)
$$

When $m \rightarrow 1$, the gamma distribution reduces to an exponential distribution. Note that the PDF in Eq. (4) can equivalently be expressed in terms of its moment generating function (MGF), which is closely related to the Laplace transform of the PDF $p_{\gamma}(\gamma)$, and defined as the expected value of $\exp (s \gamma)$ :

$$
\begin{aligned}
M_{\gamma}(s) & =\int_{0}^{\infty} d \gamma \exp (s \gamma) p_{\gamma}(\gamma) \\
& =\left(1-s \frac{\gamma_{0}}{m N}\right)^{-m}=\left(1-s \frac{\gamma_{0}}{m} \overline{\alpha^{2}}\right)^{-m}
\end{aligned}
$$

The MGF is a useful tool for analyzing average error probability in systems with fading.

Also, it can be shown that the moments of the $m$-distribution are given by

$$
\overline{\gamma^{k}}=\frac{\Gamma(m+k)}{m^{k} \Gamma(m)} \bar{\gamma}^{k}
$$

which yields, by using the average SNR, an expression for $m$ :

$$
\frac{1}{m}=\frac{\overline{\sigma_{\gamma}^{2}}}{\bar{\gamma}^{2}}=\frac{\overline{\alpha^{4}}}{{\overline{\alpha^{2}}}^{2}}-1 .
$$

Since $\alpha_{r}$ and $\alpha_{i}$ can be considered jointly normal random variables, it is possible to relate high-order moments to the lower order moments, and replace the fourth-order moment in Eq. (7) by $\overline{\alpha^{4}}=3{\overline{\alpha^{2}}}^{2}-2 \bar{\alpha}^{4}$. This yields

$$
\frac{1}{m}=2-2\left(\frac{\bar{\alpha}^{2}}{\overline{\alpha^{2}}}\right)^{2}
$$

The parameter $m$, by characterizing the amount of fading through the normalized SNR $\gamma$ variance, gives a complete description of the turbulence fading. When $m \rightarrow 1$ and the number of contributing coherent areas is large, the normalized variance is unity, as expected for a Rayleigh distribution. When $m \rightarrow \infty$, and a very small number of contributing terms add together, the normalized variance decreases. Now, the density function becomes highly peaked around the mean value $\bar{\gamma}=$ $\gamma_{0} / N$ and there is only weak fading to be considered.

To characterize turbulence effects, it is useful to develop procedures to compute $m$ and $N$. Equivalently, as Eqs. (3) and (8) describe the fading parameters $m$ and $N$ in terms of first and second moments of the fading amplitude $\alpha$, we would like to derive closed-form expressions for $\bar{\alpha}$ and $\overline{\alpha^{2}}$. In order to assess the impact of turbulence, both log-amplitude and phase fluctuations should be considered. In Eq. (1), the incident complex field in the pupil plane is expressed as

$$
E_{i}(\mathbf{r})=\exp [\chi(\mathbf{r})-j \phi(\mathbf{r})]
$$

where $\chi(\mathbf{r})$ and $\phi(\mathbf{r})$ represent the log-amplitude fluctuations (scintillation) and phase variations (aberrations), respectively, introduced by atmospheric turbulence. Also, the propagating mode of a single-mode fiber is well approximated by an un-truncated Gaussian function [15] and the fiber-mode profile referred to the receiving aperture describing the local oscillator $E_{m}(\mathbf{r})$ can be given by

$$
E_{m}(\mathbf{r})=\sqrt{\frac{2}{\pi}} \frac{1}{\omega_{m}} \exp \left[-\left(\frac{r}{\omega_{m}}\right)^{2}\right],
$$

where $\omega_{m}$ is the fiber-mode field radius at the front surface of the receiving lens. Bringing the averaging operator into Eq. (1), we find the mean fading amplitude

$$
\begin{aligned}
\bar{\alpha}= & \frac{4}{\pi D^{2}} \overline{\int d \mathbf{r} W(\mathbf{r}) E_{i}(\mathbf{r}) E_{m}(\mathbf{r})} \\
= & \frac{4}{\pi D^{2}} \exp (\bar{\chi}) \int d \mathbf{r} W(\mathbf{r}) E_{m}(\mathbf{r}) \\
& \times \overline{\exp [\chi(\mathbf{r})-\bar{\chi}] \exp [-j \phi(\mathbf{r})]},
\end{aligned}
$$

where we have assumed independence of $\chi$ and $\phi$, and extracted the mean log amplitude $\bar{\chi}$ out of the integral. We point out that because $\alpha$ results from atmospheric turbulence, we can consider phases $\phi$ that obey homogeneous, isotropic, 
zero-mean Gaussian statistics. Subject to this assumption, and the expressions for the mean of exponential functions of Gaussian variables, we can write

$$
\overline{\exp (-j \phi)}=\exp \left(-\frac{1}{2} \sigma_{\phi}^{2}\right)
$$

where the phase variance $\sigma_{\phi}^{2}$ is often used to characterize the statistics of the phase aberrations caused by atmospheric turbulence considering a Kolmogorov spectrum of turbulence [16]:

$$
\sigma_{\phi}^{2}=1.0299\left(\frac{D}{r_{0}}\right)^{5 / 3}
$$

The coefficient 1.0299 in the phase variance $\sigma_{\phi}^{2}$ assumes that no terms are corrected, e.g., by active modal phase compensation. In Eq. (13), the receiver aperture diameter $D$ is normalized by the wavefront coherence diameter $r_{0}$, which describes the spatial correlation of phase fluctuations in the receiver plane [10]. If it is also assumed that the log amplitudes $\chi$ are normal random variables [17], we can use energy conservation and the mean of exponential functions of normal variables to obtain classical results for the log-amplitude and amplitude means:

$$
\frac{\bar{\chi}=-\sigma_{\chi}^{2}}{\exp (\chi-\bar{\chi})}=\exp \left(\frac{1}{2} \sigma_{\chi}^{2}\right) .
$$

The irradiance $\beta \equiv \exp 2\left(\chi_{k}-\bar{\chi}\right)$ has a mean given by $\exp \left(2 \sigma_{\chi}^{2}\right)$. The log-amplitude variance $\sigma_{\chi}^{2}$ is often expressed as a scintillation index $\sigma_{\beta}^{2}=\exp \left(4 \sigma_{\chi}^{2}\right)-1$. Substituting Eqs. (12) and (14) into Eq. (11), and integrating the Gaussian fiber mode $E_{m}(\mathbf{r})$ over the receiving aperture, yields

$$
\bar{\alpha}=\frac{1-\exp \left(-\tau^{2}\right)}{\tau^{2}} \exp \left(-\frac{1}{2} \sigma_{\chi}^{2}\right) \exp \left(-\frac{1}{2} \sigma_{\phi}^{2}\right) .
$$

Here, the truncation parameter of the pupil $\tau \equiv D / 2 \omega_{m}$ describes the ratio of the receiver aperture diameter to the diameter of the backpropagated fiber mode. A large value of $\tau$ or $D \gg 2 \omega_{m}$ corresponds to a narrow Gaussian mode or a weakly truncated pupil. A uniformly illuminated pupil is obtained by letting $\tau \rightarrow 0$.

An explicit expression for the mean-square value

$$
\begin{aligned}
\overline{\alpha^{2}}= & \left(\frac{4}{\pi D^{2}}\right)^{2} \overline{\int d \mathbf{r} W(\mathbf{r}) E_{S}(\mathbf{r}) E_{M}(\mathbf{r})} \\
& \times \overline{\int d \mathbf{r}^{\prime} W\left(\mathbf{r}^{\prime}\right) E_{S}^{*}\left(\mathbf{r}^{\prime}\right) E_{M}^{*}\left(\mathbf{r}^{\prime}\right)}
\end{aligned}
$$

can be found by writing the two integrals as a double integral and bringing the averaging operator inside the double integral:

$$
\begin{aligned}
\overline{\alpha^{2}}= & \left(\frac{4}{\pi D^{2}}\right)^{2} \iint d r d r^{\prime} W(r) W\left(r^{\prime}\right) E_{m}(r) E_{m}^{*}\left(r^{\prime}\right) \\
& \times \overline{E_{i}(r) E_{i}^{*}\left(r^{\prime}\right)} .
\end{aligned}
$$

By changing the variables of integration from $\mathbf{r}$ and $\mathbf{r}^{\prime}$ to $\rho=\mathbf{r}-\mathbf{r}^{\prime}$ and $\mathbf{R}=\left(\mathbf{r}+\mathbf{r}^{\prime}\right) / 2$, and recognizing
$\mu(\rho)=\overline{E_{S}(\mathbf{R}+\rho / 2) E_{S}^{*}(\mathbf{R}-\rho / 2)}$ as the spatial coherence function describing the average spatial correlation length of field fluctuations, we obtain

$$
\overline{\alpha^{2}}=\frac{4}{\pi D^{2}} \frac{1-\exp \left(-2 \tau^{2}\right)}{2 \tau^{2}} \int d \rho K_{D}(\rho) \mu(\rho) .
$$

Here, $K_{D}(\rho)$ is the optical transfer function of a Gaussian pupil, i.e., the fractional area of two overlapping Gaussian beams, truncated by uniform pupils as described by the truncation parameter $\tau$, and whose centers are displaced a distance $\rho$ [18]:

$$
\begin{aligned}
K_{D}(\rho)= & \frac{4}{\pi D^{2}} \int d \mathbf{R} W\left(\mathbf{R}+\frac{1}{2} \rho\right) W\left(\mathbf{R}-\frac{1}{2} \rho\right) \\
& \times E_{m}\left(\mathbf{R}+\frac{1}{2} \rho\right) E_{m}^{*}\left(\mathbf{R}-\frac{1}{2} \rho\right) \\
= & \frac{8 \tau^{2} \exp \left(-2 \tau^{2}(\rho / D)^{2}\right)}{\pi\left[1-\exp \left(-2 \tau^{2}\right)\right]} \int_{0}^{\sqrt{1-(\rho / D)^{2}}} d q \\
& \times \exp \left(-2 \tau^{2} q^{2}\right) \int_{0}^{\sqrt{1-q^{2}}-\rho / D} d p \exp \left(-2 \tau^{2} p^{2}\right)
\end{aligned}
$$

with $\rho \leq D$. Letting $\tau \rightarrow 0$ yields the classical optical transfer function for a uniform pupil described by the fractional overlap area of two circles:

$$
K_{D}(\rho)=\frac{2}{\pi}\left\{a \cos \left(\frac{\rho}{D}\right)-\left(\frac{\rho}{D}\right)\left[1-\left(\frac{\rho}{D}\right)^{\frac{1}{2}}\right]\right\} .
$$

Equation (18) describes the fading mean-square value in terms of the field fluctuation coherence function $\mu(\rho)$. Both phase and amplitude fluctuations are considered in the field coherence. Exact expressions for $\overline{\alpha^{2}}$ can be found only when the correlation function $\mu(\rho)$ is specified. In atmospheric turbulence, based on the fact that the statistics of phase and log amplitude are homogeneous, isotropic, and Gaussian, we have [10]

$$
\begin{aligned}
\mu(\rho)= & \overline{\exp \left\{\left[\chi\left(\mathbf{R}+\frac{1}{2} \rho\right)+\chi\left(\mathbf{R}-\frac{1}{2} \rho\right)\right]\right.} \\
& \left.-j\left[\phi\left(\mathbf{R}+\frac{1}{2} \rho\right)-\phi\left(\mathbf{R}-\frac{1}{2} \rho\right)\right]\right\} \\
= & \exp \left[-\frac{1}{2} D_{W}(\rho)\right]
\end{aligned}
$$

Now, based on the Kolmogorov theory of turbulence, the wave structure function $D_{W}(\rho)$ describes the statistics of optical field variation in the atmosphere in terms of the coherence diameter $r_{0}$ as

$$
D_{W}(\rho)=6.88\left(\frac{\rho}{r_{0}}\right)^{\frac{5}{3}}
$$

By substituting Eqs. (19) and (21) into Eq. (18), we obtain an explicit expression for the fading $\overline{\alpha^{2}}$. We note that the integrand is an isotropic function of $\rho$ so that the angular part 
of the $\rho$ integration in Eq. (18) can be performed readily:

$$
\overline{\alpha^{2}}=\frac{4}{\pi D^{2}} \frac{1-\exp \left(-2 \tau^{2}\right)}{2 \tau^{2}} 2 \pi \int_{0}^{D} \rho d \rho K_{D}(\rho) \mu(\rho) .
$$

Note that the single integral in Eq. (23) can be characterized as a coherent effective area $A_{\text {eff }}$ of field fluctuations, in which case the value of $\overline{\alpha^{2}}$ decreases as the ratio of the receiver measurement area to this $A_{\text {eff }}$. Thus the inverse $N=1 / \overline{\alpha^{2}}$, used in the PDF expressions Eqs. (2) and (4), can be interpreted as the average number of field coherent areas (spatial modes of the field pattern) over the measurement area. The exact number of spatial modes in the field pattern affecting the measurement area depends on the field spatial coherence and the area of integration of the aperture. In the case of a receiver area that is very large compared with the average correlation length of the field fluctuations, the receiver aperture function $K_{D}(\rho)$ is much wider than the autocorrelation function $\mu(\rho)$. We can factor out $K_{D}(\rho=0)=1$ from the integral in Eq. (23) which, using Eqs. (21) and (22), can be solved in a closed form to obtain

$$
\overline{\alpha^{2}}=\frac{1-\exp \left(-2 \tau^{2}\right)}{2 \tau^{2}} 1.09\left(\frac{r_{0}}{D}\right)^{2} \Gamma\left[\frac{6}{5}, 1.08\left(\frac{D}{r_{0}}\right)^{5 / 3}\right] .
$$

Here, $\Gamma(a, x)$ is the lower incomplete gamma function. If the receiver aperture is much larger than the coherence diameter $r_{0}$, i.e., $D \gg r_{0}$, and we let $\tau \rightarrow 0$ to obtain a uniform illuminated pupil, Eq. (24) simplifies to $\Omega=1.007\left(r_{0} / D\right)^{2}$. To a good approximation, the aperture can be considered to consist of $N \approx\left(D / r_{0}\right)^{2}$ independent cells, each of diameter $r_{0}$. On the other hand, when the field correlation area is much wider than the aperture area, in Eq. (23) we can simply assume $\mu(\rho)=1$, and in this limit the number of field coherent areas within the measurement area approaches unity.

We can extend this analysis of the atmospheric coherent fading and consider, along with the first-order statistical properties $\bar{\alpha}$ and $\overline{\alpha^{2}}$, some higher order statistics. Of special interest to our study are the joint properties of the fading amplitudes $\alpha_{k}$ and $\alpha_{l}$ collected by two different apertures centered at separated points in space. It is simple to show that, given two apertures with centers separated by a distance $\Delta r$, the fading correlation function $C_{\alpha}(\Delta r)=\overline{\alpha_{k} \alpha_{l}^{*}}$ is given by

$$
\begin{aligned}
C_{\alpha}(\Delta r)= & \frac{4}{\pi D^{2}} \frac{1-\exp \left(-2 \tau^{2}\right)}{2 \tau^{2}} \\
& \times \int_{0}^{2 \pi} d \phi \int_{0}^{D / 2} \rho d \rho K_{D}(\rho) \\
& \times \mu\left\{\left[(\Delta r+\rho \cos \phi)^{2}+(\rho \sin \phi)^{2}\right]^{1 / 2}\right\} .
\end{aligned}
$$

The superscript ${ }^{*}$ represents the conjugation operator. As expected from the definition of the correlation function, when $\Delta r=0$, we have $C_{\alpha}(\Delta r=0)=\overline{\alpha^{2}}$, as described in Eq. (23). This result is used in the next section to describe the degree of correlation among the different fading signals combined by a coherent fiber array.

By substituting Eqs. (15) and (23) into Eqs. (3) and (8), we can express the Nakagami- $m$ parameters $N$ and $m$ in terms of two well-known magnitudes in atmospheric turbulence studies: the log-amplitude variance $\sigma \chi^{2}$, which is often used to characterize the statistics of amplitude fluctuations, and the wavefront coherence diameter $r_{0}$, used to describe the spatial correlation of phase fluctuations in the receiver plane.

\section{Performance Analysis of a Closely Packed HEXAGONAL COHERENT ARRAY}

In a field conjugation fiber array, the downconverted electrical signals at the output of the single-mode fibers need to be adaptively co-phased and scaled before they are summed, to lessen fading caused by atmospheric turbulence and compensate for imperfect fiber coupling efficiency (see Fig. 1). That is equivalent to maximal-ratio combining (MRC) of the signals from the array subapertures, considering them to be branches of a diversity receiver [19]. MRC diversity schemes assume perfect knowledge of the branch amplitudes and phases, and require that the individual signals from the branches be weighted by their signal amplitude to noise power ratios, and then summed coherently. Thus, assuming equal noise powers in each array element, a receiver should weight individual signals by the complex conjugates of their respective fading gains, and then add them (hence the name "field conjugation array"). As in any system using coherent MRC, the instantaneous SNR $\gamma_{T}$ of the combined signals is the ratio of the power of the coherent combination of the individual signals to the power of the incoherent combination of the individual noises. If an optimum voltage gain proportional to the signal amplitude is assumed for each element in the array, and if equal noise powers are assumed, the resultant composite SNR for an $L$-element coherent fiber array is

$$
\gamma_{T}=\sum_{l=1}^{L} \gamma_{l}=\gamma_{0} \sum_{l=1}^{L} \alpha_{l}^{2}
$$

Therefore, the instantaneous array combiner SNR $\gamma_{T}$ is the sum of the $\gamma_{l}$, the array element SNRs. For independent subaperture signals and equal average branch SNRs, i.e., $\bar{\gamma}_{l}=$ $\bar{\gamma}$ for all $l \in\{1,2, \ldots, L\}$, the PDF of the received SNR $\gamma_{T}$ at the output of a perfect $L$-branch coherent array can be described as a sum of $L$ independent and identically distributed gamma random variables. Thus, the random variable $\gamma_{T}$ is also described by the gamma distribution Eq. (4) with a shape parameter $(m L)$. However, for close coherent fiber array receivers, with insufficient collecting aperture spacing, it is not realistic to assume that the combined signals are independent of one another. In this scenario, the degree of correlation among the different fading coefficients $\alpha_{l}$ describing $\gamma_{T}$ in Eq. (26) will depend on several factors, including atmospheric conditions and the exact geometry of the coherent array receiver.

Conventional analysis of a coherent array in correlated fading is complicated as it is difficult to obtain closed-form expressions quantifying performance. To facilitate the analysis, we can transform the dependent fading coefficients $\alpha_{l}$ into a new set of independent virtual components and express the array SNR as a linear function of independent virtual variables. We need to define a transformation of the $L$ coefficients $\alpha_{l}$ that will preserve the total intensity 
$\sum \alpha_{l}^{2}$ in Eq. (26) but eliminate the correlation between the various fading components. Transforming the dependent physical branch variables into a new set of independent virtual branches allows us to express the array output SNR as a linear combination of the SNRs at the independent virtual branches [20,21].

Let $\alpha$ be the $L \times 1$ vector defined by

$$
\alpha=\left[\alpha_{1}, \alpha_{2}, \ldots, \alpha_{L}\right]^{T}
$$

and define a correlation matrix $\mathbf{C}_{\alpha}$ by

$$
\mathbf{C}_{\alpha}=\overline{\alpha \alpha^{\dagger}}
$$

where the superscripts $T$ and $\dagger$ represent the transpose and the conjugate-transpose operators, respectively. Thus, the element $C_{k, l} \equiv \overline{\alpha_{k} \alpha_{l}^{*}}$ of the correlation matrix $\mathbf{C}_{\alpha}$ is given by Eq. (25), with $\Delta r$ defining the distance between the elements $k$ and $l$ in the array. The correlation matrix $\mathbf{C}_{\alpha}$ is Hermitian, i.e., conjugate-symmetric above and below the diagonal and, consequently, there exists a linear $L \times L$ transformation matrix $\mathbf{L}_{0}$ that diagonalizes $\mathbf{C}_{\alpha}$ :

$$
\mathbf{C}_{\alpha}^{\prime}=\mathbf{L}_{0} \mathbf{C}_{\alpha} \mathbf{L}_{0}^{\dagger}
$$

This linear transformation of the fading correlation matrix results in a new diagonal matrix $\mathbf{C}_{\alpha}^{\prime}$ and decorrelates the fading coefficients $\alpha_{l}$. Also, because the diagonalizing transformation is unitary, i.e., $\mathbf{L}_{0} \mathbf{L}_{0}^{\dagger}$ equals the identity matrix, this transformation is lossless and the sum of the diagonal elements of the correlation matrix remains unchanged:

$$
\sum_{i=1}^{L} \overline{\alpha_{l}^{2}}=\sum_{i=1}^{L} \lambda_{l}
$$

Here, the $\lambda_{l}$ represent the eigenvalues of the matrix $\mathbf{C}_{\alpha}$, and characterize the fading intensities of the new fading components after the diagonalizing transformation. If Eq. (30) is used in Eq. (26), we see that the composite array SNR $\gamma_{T}$ is transformed from a sum of $L$ correlated SNRs, $\gamma_{0} \alpha_{l}^{2}$, to an equivalent sum of $L$ uncorrelated, independent SNRs, $\gamma_{0} \lambda_{l}$. Hence, the MGF for the correlated Nakagami fading can be expressed as the product of the MGFs associated with individual array elements:

$$
M_{T}(s)=\prod_{l=1}^{L} M_{l}(s)=\prod_{l=1}^{L}\left(1-s \frac{\gamma_{0}}{m} \lambda_{l}\right)^{-m} .
$$

Here, $M_{l}(s)$ denotes the MGF Eq. (5) for the $l$ th virtual SNR. It is worth mentioning that the evaluation of most common performance measures of a coherent communication system can be accomplished based entirely on knowledge of the MGF of the output SNR, without ever having to compute its PDF. We will use $M_{T}(s)$, given by Eq. (31), to estimate the performance of the MRC coherent fiber array.

Perhaps the best and simplest performance measures of a communication system in the presence of fading are the average SNR $\bar{\gamma}$ and the normalized SNR variance $\sigma_{\gamma}^{2} / \bar{\gamma}^{2}$. The normalized variance (or, equivalently, the normalized standard deviation) describes the inherent statistical uncertainty of the SNR $\gamma$ caused by turbulence. In order to estimate these two performance measures, we must know at least the first statistical moments of the instantaneous SNR $\gamma$. If $M_{T}(s)$ is differentiable at zero, then the $n$th moment about the origin is given by the $n$th derivative $\overline{\gamma^{n}}=M_{T}^{(n)}(0)$. The mean and variances are therefore

$$
\begin{aligned}
& \bar{\gamma}=M_{T}^{\prime}(0), \\
& \sigma_{\gamma}^{2}=M_{T}^{\prime \prime}(0)-\left[M_{T}^{\prime}(0)\right]^{2} .
\end{aligned}
$$

By using finite differences of several orders, it is straightforward to perform numerical differentiation of the numerical moment generating function given by Eq. (31).

Perhaps the most important performance metric is the average symbol error probability (SEP). We can apply the AWGN SEP expression [22]

$$
p_{s}(E \mid \gamma) \approx \frac{a}{\pi} \int_{0}^{\pi / 2} d \phi \exp \left(-\frac{b \gamma}{\sin ^{2} \phi}\right)
$$

to determine the performance over the atmospheric fading channel. Here, $a$ and $b$ depend on the modulation format. For the $M$-ary coherent modulation PSK that will be considered in this analysis, we have $a=2$ and $b=\sin ^{2}(\pi / M)$. When atmospheric fading is present, the received SNR $\gamma$ varies randomly and, consequently, the $\operatorname{SEP} p_{s}(E \mid \gamma)$ conditioned on the SNR $\gamma$ is also random. Various performance metrics depend on the rate of change of the fading and on the average SEP. The unconditional SEP $p_{s}(E)$ of our ideal coherent array receiver in the presence of atmospheric fading can be obtained by averaging the AWGN conditional SEP $p_{S}(E \mid \gamma)$ over the PDF $p_{T}(\gamma)$ of the instantaneous fading SNR at the output of the coherent fiber array [23]:

$$
\begin{aligned}
p_{S}(E) & =\int_{0}^{\infty} d \gamma p_{S}(E \mid \gamma) p_{T}(\gamma) \\
& =\frac{a}{\pi} \int_{0}^{\pi / 2} d \phi M_{T}\left(-\frac{b}{\sin ^{2} \phi}\right)
\end{aligned}
$$

where we have interchanged the order of integration and made use of the definition of the MGF. This MGF-based approach is quite useful in simplifying our analysis. Although the integration of Eq. (34) cannot be carried out in closed form, we are able to carry out the integration in Eq. (34) using a simple Gaussian-Legendre quadrature formula which, along with the numerical evaluation of the MGF $M_{T}(s)$ Eq. (31), yields high accuracy.

Figure 2 considers the mean and standard deviation of the output SNR of field conjugation adaptive arrays, as given by Eq. (34). We consider an $L$-element array and assume that the subapertures are arranged in a hexagonal close-packed array, as shown in Fig. 1. The larger circle represents a single receiver aperture of diameter $D$. The small circles represent packed subapertures. Each subaperture contains a lens that couples the received light into a single-mode fiber. For comparison of the receiver performance between an $L$-element array and a single large aperture, we force the hexagonal array to be packed within the limits of the single aperture area. The hexagonal distribution is the densest way to arrange circles 

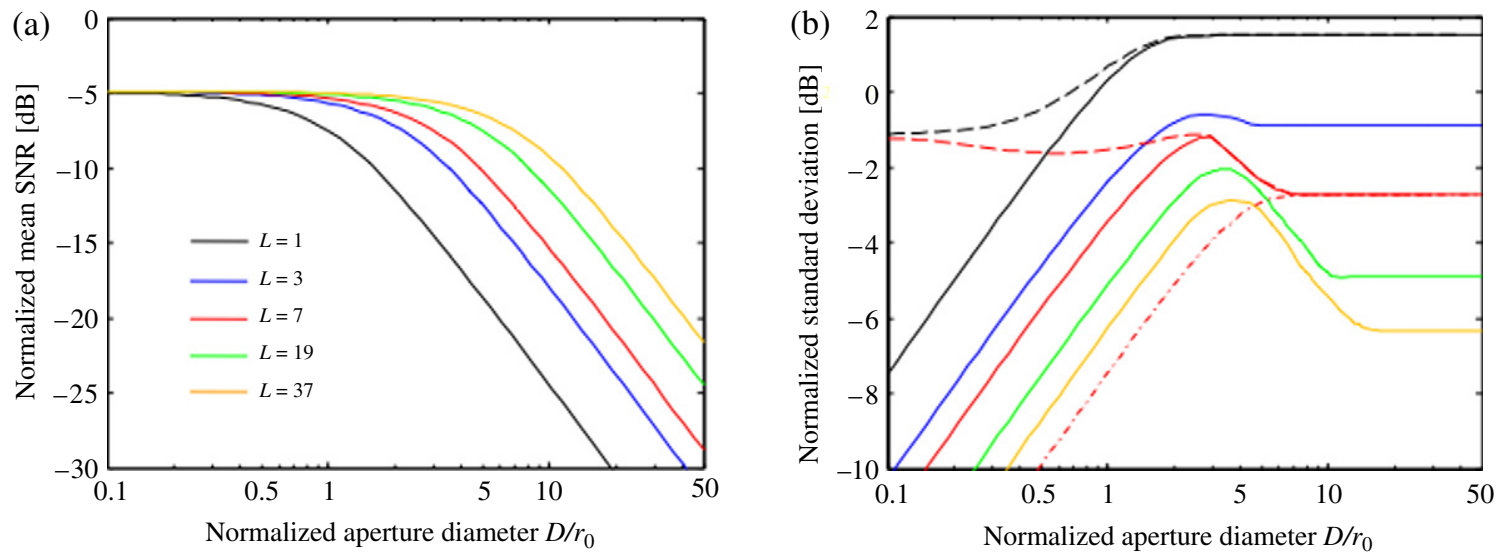

Fig. 2. (Color online) Normalized (a) mean and (b) standard deviation of SNR versus normalized receiver aperture diameter $D / r_{0}$. The performance is shown for different numbers of subapertures $L$ in a closely packed hexagonal coherent array. The case $L=1$ corresponds to a monolithic aperture (black line). When a single aperture is considered, $D$ describes the receiver aperture diameter. For comparison of the receiver performance between an $L$-element array and a single large aperture, we force the hexagonal array to be packed within the limits of the single aperture diameter $D$. The analysis takes account of the array fill factor and considers a coupling-geometry parameter $\tau$ for each subaperture lens equal to 1.12. Solid lines indicate that amplitude fluctuations are neglected by assuming $\sigma_{\beta}^{2}=0$. Dashed lines indicate that amplitude fluctuations are considered, assuming a scintillation index $\sigma_{\beta}^{2}=1$. In (b), the red dot-dashed line indicates the case of $L=7$ subapertures with statistically independent fading.

in a plane. Still, note that each subaperture will have a pupil area slightly smaller than $1 / L$ times the pupil area of the single receiver system and an array fill factor needs to be considered in our analysis. For instance, for the array with $L=7$ elements in Fig. 1, the array fill factor is $7 / 9$. Also, the truncation parameter $\tau=D / 2 \omega_{m}$, describing the coupling geometry of the array, must be chosen to optimize the receiving system performance. Although the optimal truncation parameter may depend on the level of atmospheric turbulence considered, this dependency is very weak, and it is reasonable to choose a value $\tau_{\text {opt }}=1.12$, which is optimal in the absence of turbulence, when the incident plane wave is fully coherent [24]. The same value of the truncation parameter $\tau$ is used for each subaperture lens and for the single, larger lens.

We study the mean SNR $\bar{\gamma}$ in Fig. 2(a) and the SNR normalized standard deviation $\sigma_{\gamma} / \bar{\gamma}$ in Fig. 2(b) as a function of several parameters: the average turbulence-free SNR $\gamma_{0}$, the receiver aperture diameter $D$, the number of subapertures $L$ of the array, and the strength of atmospheric turbulence. Turbulence is quantified by two parameters: the phase coherence length $r_{0}$ and the scintillation index $\sigma_{\beta}^{2}$. The value of the scintillation index $\sigma_{\beta}^{2}=1$ corresponds to strong scintillation, but still below the saturation regime. When we assume no scintillation, $\sigma_{\beta}^{2}=0$, the effect of turbulence is simply to reduce the coherence length $r_{0}$. For a fixed coherent diameter $r_{0}$, as the aperture diameter $D$ is increased, the normalized aperture diameter $D / r_{0}$ increases, and turbulence reduces the photo-electric downconversion efficiency.

In Fig. 2(a), the mean SNR is plotted against the normalized aperture diameter $D / r_{0}$ for different numbers of subapertures $L$ in the hexagonal array. The SNR is expressed in $\mathrm{dB}$, referenced to the turbulence-free SNR $\gamma_{0}$. This corresponds to the mean intensity fading $\overline{\alpha^{2}}=1 / N$, according to the relationship $\gamma=\gamma_{0} \alpha^{2}$. Note that the mean SNR is just a representation of the fiber coupling efficiency. The received signal beam must be coupled into a single-mode fiber, but atmospheric turbulence degrades the spatial coherence of a laser beam and limits the fiber coupling efficiency and, consequently, the available mean SNR at the output of each fiber in the array. As expected for one single monolithic aperture $L=1$, if $D$ is less than $r_{0}$, the normalized mean SNR $\bar{\gamma} / \gamma_{0}$ remains constant. A truncation parameter $\tau=1.12$ reduces the mean SNR by $5 \mathrm{~dB}$. When the diameter $D$ is larger than $r_{0}$, atmospheric turbulence limits the effective receiving aperture to the dimensions of the coherence diameter $r_{0}$ and the normalized mean SNR goes down very quickly. When the normalized aperture diameter $D / r_{0}$ is large, an increase in the number of hexagonal array subapertures improves the situation significantly. For instance, when a large normalized aperture $D / r_{0}=10$ is considered, increasing the number of subapertures $L$ from 1 to 19 increases the mean SNR by more than $13 \mathrm{~dB}$. The mean SNR is just a representation of the fiber coupling efficiency; the advantage of a fiber array in terms of the mean SNR and the fiber coupling efficiency is that the number of field coherence areas $N$ over each subaperture is smaller than it would be over a single large aperture.

In Fig 2(b), we plot the normalized SNR standard deviation (SNR uncertainty or relative error) $\sigma_{\gamma} / \bar{\gamma}$ against the normalized aperture diameter $D / r_{0}$ for different values of the number of subapertures $L$ in the hexagonal array. In the limit of weak turbulence (small normalized aperture diameter $D / r_{0}$ ), the normalized variance tends asymptotically to 0 . When $L=1$, the SNR standard deviation reaches a maximum value of almost $1.6(2 \mathrm{~dB})$. Once again, an increase in the number of array subapertures will improve the situation and decrease the SNR uncertainty. As an example, when the normalized aperture is $D / r_{0}=10$, increasing the number of subapertures $L$ from 1 to 19 decreases the SNR uncertainty by roughly $6 \mathrm{~dB}$, from $1 \mathrm{~dB}$ to $-5 \mathrm{~dB}$. As we observe in Fig. 2(b), the effects of scintillation are noticeable for small aperture diameters and must be properly considered. For relatively small apertures, amplitude 

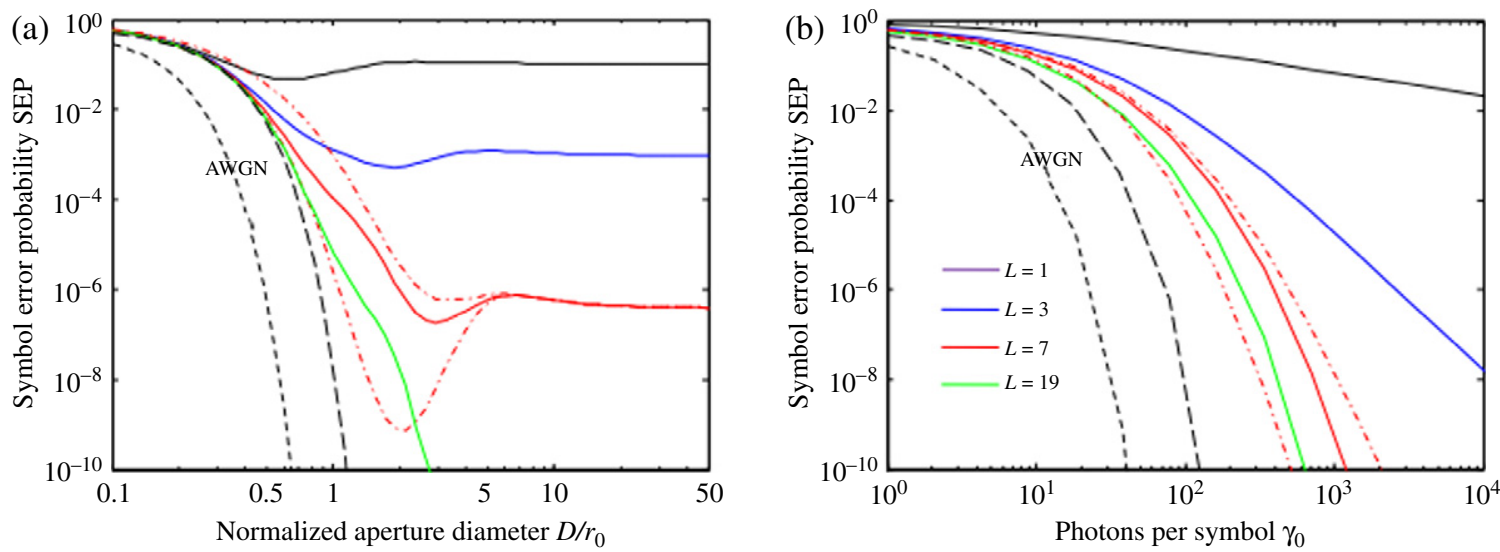

Fig. 3. (Color online) SEP versus (a) normalized receiver aperture diameter $D / r_{0}$ and (b) number of photons per symbol for QPSK with coherent detection and AWGN. The performance is shown for different numbers of subapertures $L$ in the closely packed hexagonal coherent array. The case $L=1$ corresponds to a monolithic aperture (black line). When a single aperture is considered, $D$ describes the receiver aperture diameter. For comparison of the receiver performance between an $L$-element array and a single large aperture, we force the hexagonal array to be packed within the limits of the single aperture diameter $D$. The analysis considers the array fill factor and considers a coupling-geometry parameter for each subaperture lens of $\tau=1.12$. In (a), the turbulence-free SNR per symbol $\gamma_{0}$ is proportional to the square of the aperture diameter $D$. For the smallest aperture considered, we assume $\gamma_{0}=0 \mathrm{~dB}$. In (b), the normalized aperture diameter is set to $D / r_{0}=2$. Solid lines indicate that amplitude fluctuations are neglected by assuming $\sigma_{\beta}^{2}=0$. The upper red dot-dashed line indicates the case $L=7$ considering scintillation, with scintillation index $\sigma_{\beta}^{2}=1$. The lower red dot-dashed line indicates the case $L=7$, considering scintillation, and neglecting fading correlation. Black dashed lines indicate the no-turbulence AWGN case (Eq. (33)). Black dotted lines indicate the no-turbulence AWGN case with $\tau=0$, corresponding to a uniformly illuminated pupil, which corresponds to a 5-dB mean SNR improvement over the case $\tau=1.12$.

scintillation is dominant, and the normalized variance is virtually unaffected by wavefront phase distortions. When the aperture is larger, phase distortion becomes dominant and the scintillation index $\sigma_{\beta}^{2}$ is of little relevance in the SNR uncertainty. It is also interesting to consider the case of subapertures with statistically independent fading. Such independent signals could be obtained by increasing the spacing between subapertures in the array. If the output of a perfect $L$-element combiner in the atmosphere were to be described as a sum of $L$ independently fading signals, the output SNR uncertainty would be expected to be reduced. That is the case in the limit of weak turbulence or small normalized apertures; for example, when 7-element arrays are considered, the uncertainty is almost $4 \mathrm{~dB}$ smaller for ideal independent subapertures. This 4- $\mathrm{dB}$ difference is the penalty for using closely packed arrays that combine highly correlated signals. However, when strong turbulence or large normalized apertures are considered, the field coherence areas are smaller than any array subaperture considered in this analysis and the signals collected by the different subapertures are very nearly uncorrelated. In this regime, the multiple replicas combined at the array receiver are already statistically independent, and spreading apart the elements of the array would not reduce the SNR uncertainty.

Figure 3 presents the SEP given by Eq. (34) for an $L$-element array receiver. Figure 3(a) shows the SEP versus the normalized aperture diameter $D / r_{0}$, while Fig. 3(b) shows the SEP versus the turbulence-free SNR $\gamma_{0}$. In Fig. $3(\mathrm{a})$, for the smallest aperture diameter considered, the turbulence-free SNR has a value $\gamma_{0}=0 \mathrm{~dB}$. For any other aperture diameter, the value of $\gamma_{0}$ is proportional to $D^{2}$. When we assume no scintillation, $\sigma_{\beta}^{2}=0$, the effect of turbulence is simply to reduce the coherence length $r_{0}$. For a single large aperture, even using a relatively small normalized aperture diameter $D / r_{0}=1$, turbulence introduces a large SEP penalty. When multi-aperture array receivers are considered, in most situations a substantial performance improvement is obtained. An array with just $L=19$ subapertures yields significant improvement for even the largest normalized apertures considered. The performance of such array receivers is very close to the performance expected in an AWGN system. In Fig. 3(a), when large normalized apertures $D / r_{0}$ are considered, the SEP becomes independent of the scintillation index $\sigma_{\beta}^{2}$, and tends toward an asymptotic value that is independent of the normalized aperture diameter $D / r_{0}$.

Figure 3(b) shows the SEP performance for different values of $L$, the number of array elements. Even using a relatively small normalized aperture diameter $D / r_{0}=2$, when a single large aperture receiver is used, turbulence introduces more than a $30-\mathrm{dB}$ performance penalty at $10^{-3}$ SEP. When multi-element arrays are used, the performance improves markedly. For instance, considering a small array receiver with $L=7$ subapertures at $\mathrm{SEP}=10^{-3}$, the SNR penalty is just below $10 \mathrm{~dB}$. This value should be contrasted with the 6 - $\mathrm{dB}$ penalty observed in Fig. 3(b) when $L=7$ independently fading signals are ideally combined. Also, although these results assume no scintillation, when we impose a strong scintillation index of $\sigma_{\beta}^{2}=1$, the penalty increase is less than $1 \mathrm{~dB}$ at $\mathrm{SEP}=10^{-3}$.

\section{CONCLUSIONS}

We have numerically evaluated the performance of adaptive field conjugation array receivers in coherent laser communications through the turbulent atmosphere. We analyzed 
coherent fiber arrays consisting of densely packed multiple subapertures in a hexagonal arrangement, considering the effects of log-normal amplitude fluctuations and Gaussian phase fluctuations, in addition to local oscillator shot noise.

By noting that the impact of atmospheric turbulence on coherent receivers can be statistically described by a Nakagami- $m$ distribution, our model takes into account fundamental principles of atmospheric propagation without requiring a detailed description of the instantaneous realization of atmospheric turbulence. Such a specification is difficult because of the inherent complexity of the propagation problem. Our formulation results in a two-parameter distribution for the return signal. In this analysis, we compute the parameters of the Nakagami- $m$ distribution using a heuristic theory.

For fiber adaptive arrays, where the atmospheric fading on the subapertures is correlated or dependent, we can solve the problem by transforming it into an independent problem. The MGF-based approach used in our analysis has provided easily evaluable analytical expressions for the signal statistical moments and the symbol error probabilities. We have used them to study the effects of various parameters on performance, including turbulence level, signal strength, receiver aperture size and the number of subapertures in the coherent fiber array. We have separately quantified the effects of amplitude fluctuations and wavefront phase distortion on system performance, and have identified different regimes of turbulence, depending on the receiver aperture diameter normalized to the coherence diameter of the wavefront phase. When the normalized aperture is larger, amplitude fluctuations become negligible, and phase fluctuations become dominant. For most typical free-space laser communication situations, using coherent arrays with a reasonably small number of subapertures such as $L=19$ increases the communication performance by several decibels.

\section{ACKNOWLEDGMENT}

The research of Aniceto Belmonte was partially funded by the Spanish Department of Science and Innovation MICINN Grant No. TEC 2009-10025.

\section{REFERENCES}

[1] G. Li, "Recent advances in coherent optical communication," Adv. Opt. Photon., vol. 1, pp. 279-307, 2009.

[2] K. Kiasaleh, "Performance of coherent DPSK free-space optical communication systems in $K$-distributed turbulence," IEEE Trans. Commun., vol. 54, pp. 604-607, 2006.

[3] T. A. Tsiftsis, "Performance of heterodyne wireless optical communication systems over Gamma-Gamma atmospheric turbulence channels," Electron. Lett., vol. 44, pp. 373-375, 2008.

[4] A. Belmonte and J. M. Kahn, "Performance of synchronous optical receivers using atmospheric compensation techniques," Opt. Express, vol. 16, pp. 14151-14162, 2008.
[5] H. G. Sandalidis, T. A. Tsiftsis, and G. K. Karagiannidis, "Optical wireless communications with heterodyne detection over turbulence channels with pointing errors," J. Lightwave Technol., vol. 27, pp. 4440-4445, 2009.

[6] E. J. Lee and V. W. Chan, "Diversity coherent and incoherent receivers for free-space optical communication in the presence and absence of interference," J. Opt. Commun. Netw., vol. 1, pp. 463-483, 2009.

[7] A. Belmonte and J. M. Kahn, "Capacity of coherent free-space optical links using diversity-combining techniques," Opt. Express, vol. 17, pp. 12601-12611, 2009.

[8] M. Niu, J. Cheng, and J. F. Holzman, "Exact error rate analysis of equal gain and selection diversity for coherent free-space optical systems on strong turbulence channels," Opt. Express, vol. 18, pp. 13915-13926, 2010.

[9] N. Cvijetic, D. Qian, J. Yu, Y.-K. Huang, and T. Wang, "Polarization-multiplexed optical wireless transmission with coherent detection," J. Lightwave Technol., vol. 28, pp. 1218-1227, 2010 .

[10] D. L. Fried, "Optical heterodyne detection of an atmospherically distorted signal wave front," Proc. IEEE, vol. 55, pp. 57-67, 1967.

[11] R. M. Gagliardi and S. Karp, Optical Communications. John Wiley \& Sons, 1995.

[12] J. W. Goodman, Speckle Phenomena in Optics. Theory and Applications. Ben Roberts \& Company, 2007.

[13] M. Nakagami, "The $m$-distribution. A general formula of intensity distribution of rapid fading," in Statistical Methods in Radio Wave Propagation, W. C. Hoffman, Ed. Pergamon Press, 1960.

[14] S. M. Aghajanzadeh and M. Uysal, "Diversity-multiplexing trade-off in coherent free-space optical systems with multiple receivers," J. Opt. Commun. Netw., vol. 2, pp. 1087-1094, 2010.

[15] J. A. Buck, Fundamentals of Optical Fibers, 2nd Ed. Wiley, 2004.

[16] R. J. Noll, "Zernike polynomials and atmospheric turbulence," J. Opt. Soc. Am., vol. 66, pp. 207-211, 1976.

[17] J. W. Strohbehn, T. Wang, and J. P. Speck, "On the probability distribution of line-of-sight fluctuations of optical signals," Radio Sci., vol. 10, pp. 59-70, 1975.

[18] V. N. Mahajan, Optical Imaging and Aberrations, Part II. Wave Diffraction Optics. SPIE Press, 2004.

[19] J. D. Parsons, "Diversity techniques in communications receivers," in Advanced Signal Processing, D. A. Creasey, Ed. Peregrinus, 1985, Chap. 6.

[20] V. A. Aalo, "Performance of maximal-ratio diversity systems in a correlated Nakagami-fading environment," IEEE Trans. Commun., vol. 43, pp. 2360-2369, 1995.

[21] M. Z. Win, G. Chrisikos, and J. H. Winters, "MRC performance for M-ary modulation in arbitrarily correlated Nakagami fading channels," IEEE Commun. Lett., vol. 4, pp. 301-303, 2000.

[22] J. G. Proakis and M. Salehi, Digital Communications. McGraw-Hill, 2007.

[23] M. K. Simon and M.-S. Alouini, "A unified approach to the performance analysis of digital communications over generalized fading channels," IEEE Proc., vol. 86, pp. 1860-1877, 1998.

[24] P. J. Winzer and W. R. Leeb, "Fiber coupling efficiency for random light and its applications to lidar," Opt. Letters, vol. 23, pp. 986-988, 1998. 